\section{Association of autonomic dysfunction and clozapine}

\author{
Heart rate variability and risk for sudden death in patients with \\ schizophrenia on long-term psychotropic medication
}

HAGIT COHEN, URI LOEWENTHAL, MICHAEL MATAR and MOSHE KOTLER

\section{Background Antipsychotic medications cause a wide range of adverse effects and have been associated with sudden death in psychiatric patients.}

\begin{abstract}
Aims To supply power spectral analysis of heart rate variability as a tool to examine the arrythmogenic effects of neuroleptics.
\end{abstract}

\begin{abstract}
Method Heart rate analysis was carried out in patients with schizophrenia on standard doses of neuroleptic monotherapy - 21 were on clozapine, 18 on haloperidol and 17 on olanzapine - and in 53 healthy subjects.

Results Patients with schizophrenia on clozapine had significantly higher heart rate, lower heart rate variability and lower high-frequency and higher lowfrequency components compared with patients on haloperidol or olanzapine and matched control subjects. Prolonged QTc intervals were more common in patients than controls.
\end{abstract}

\section{Conclusions Patients treated with neuroleptic medications, especially clozapine, showed autonomic dysregulation and cardiac repolarisation changes. Physicians should be aware of this adverse reaction.}

Declaration of interest None.
Antipsychotic medications have been associated with significant rates of cardiovascular adverse effects and instances of sudden unexplained death (Ruschena et al, 1998; Appleby et al, 2000). Retrospective studies conclude that cardiovascular mortality in psychiatric patients is higher than in the general population, especially in patients with schizophrenia treated with antipsychotic drugs (Ruschena et al, 1998; Chute et al, 1999). Disturbances of electrical conduction, especially ventricular repolarisation, have been attributed to a variety of neuroleptic drugs. Most sudden deaths of cardiac origin are attributed to ventricular tachycardia and/or fibrillation, in which the autonomic nervous system (ANS) may play an important role. One reliable method for quantifying ANS activity is the analysis of heart rate variability (HRV) (Akselrod et al, 1981). The aim of this study was to apply power spectral analysis of HRV as a tool to assess the potential arrythmogenic effects of a variety of antipsychotic medications, both classic and atypical.

\section{METHOD}

\section{Subjects}

Fifty-six patients who met DSM-IV (American Psychiatric Association, 1994) criteria for schizophrenia, as assessed by the Schedule for Affective Disorders and Schizophrenia (Endicott \& Spitzer, 1987), were recruited from the in-patient and out-patient treatment programmes at the Beer-Sheva Mental Health Center. None of the patients had a known history of cardiac abnormalities or any concomitant disease known to affect the autonomic nervous system. All patients were on neuroleptic monotherapy: 21 were treated with $300-700 \mathrm{mg} /$ day clozapine, 18 with $5-10 \mathrm{mg} /$ day haloperidol and 17 with 5$20 \mathrm{mg} /$ day olanzapine.

\section{Controls}

It proved impossible both to recruit a sufficient number of untreated patients with schizophrenia, because these are rare in Israel today, and certainly to create an age- and gender-matched control group. We therefore used a healthy control group for comparison. The 53 healthy volunteers were matched for age, gender, smoking and time of day of electrocardiogram (ECG) recordings. None had any serious or disabling coexisting diseases. Controls had not taken any psychotropic or other medications known to alter autonomic activity for at least 4 weeks prior to the study.

The Helsinki Ethics Committee of the hospital approved the study. All participants gave their written consent after having received detailed information about the study.

\section{Procedure}

Electrocardiographic recordings were performed by connecting the subjects to a Holter monitor (Oxford 4-24) in a seated position at complete rest. After allowing $10 \mathrm{~min}$ for stabilisation, a 10-min ECG recording was made. Subjects were instructed to breathe normally and the respiratory rate was monitored by a strain-gauge-based respiration transducer, wrapped around the chest and abdomen halfway between the rib cage and navel.

\section{Analysis of recordings}

Electrocardiographic data were amplified, digitised $(500 \mathrm{~Hz}$, width pass $=0.05-35 \mathrm{~Hz})$ and stored using a personal-computer-based software system. QRS complexes were classified. Premature ventricular beats, electrical 'noise' or aberrant beats were rejected. The threshold for rejection was set at $\pm 15 \%$ of the reference RR duration. The $\mathrm{R}-\mathrm{R}$ intervals between normal QRS complexes were extracted and a regularly spaced time series was sampled at $2 \mathrm{~Hz}$ with the use of a low-pass filter. Finally, we transformed the time signal into a frequency signal by using a fast Fourier transform (Task Force, 1996).

\section{Frequency domain analysis}

Two frequency bands were calculated: the low-frequency (LF) band $(0.04-0.15 \mathrm{~Hz})$, which gives a measure of sympathetic activity with some influence from the parasympathetic nervous system; and the 
high-frequency $(\mathrm{HF})$ band $(0.15-0.40 \mathrm{~Hz})$, which solely reflects parasympathetic activity. The area $\left(\mathrm{ms}^{2}\right)$ under the curve was calculated for each frequency band. There is some disagreement over the lowfrequency component. Some investigators regard it as a marker of sympathetic activity, whereas others consider it an index influenced by both sympathetic and vagal systems. To cancel out the influence of the parasympathetic activity on the lowfrequency spectral power, the LF/HF ratio was calculated. This ratio provides a measure of the sympathovagal balance, where an increase in the LF/HF ratio reflects a predominance of sympathetic over parasympathetic activity. We calculated the total variability of the heart rate oscillations in the total power spectrum $(0.01-0.4 \mathrm{~Hz})$ as an expression of the overall variability. Because total power varies greatly between individual subjects, power was determined in both absolute units and as normalised values. The power in normalised units was calculated by dividing the absolute power of a given component (area under the component curve) by the total power minus the $0-0.04 \mathrm{~Hz}$ component.

An independent observer, who was 'blind' as to whether the data referred to a patient or a control subject, performed all ECG recordings.

\section{Measurements of $\mathbf{Q T}$ interval}

The corrected QT interval was calculated by the method of Bazett $(\mathrm{QT} c=\mathrm{QT} /(\sqrt{ } \mathrm{RR}))$. The mean QT and the mean RR were used to calculate the mean QTc for each lead. Heart rate was derived from the mean of the RR intervals. The QT and RR intervals were measured manually with callipers by a single observer. The QT intervals were measured from the onset of the QRS complex to the end of the $T$ wave. The end of the $\mathrm{T}$ wave was defined as the point of return to the isoelectric line.

\section{Statistical analysis}

To compare effects between groups, the variables were analysed by analysis of covariance (one-way ANCOVA), with age and smoking status as the covariates. Age was entered as a covariate because HRV decreases with increasing age $(r=-0.81$, $\left.r^{2}=0.66 ; P<0.01\right)$. Post hoc comparisons employed Scheffé's test. Because of the skewness of the data, logarithmic transformation was performed on the absolute

Table I Demographic data of the patients with schizophrenia

\begin{tabular}{|c|c|c|c|c|}
\hline & $\begin{array}{l}\text { Patients treated } \\
\text { with clozapine } \\
\qquad(n=2 \mathrm{I}) \\
\text { I }\end{array}$ & $\begin{array}{l}\text { Patients treated } \\
\text { with haloperidol } \\
\qquad(n=18) \\
\text { II }\end{array}$ & $\begin{array}{l}\text { Patients treated } \\
\text { with olanzapine } \\
\qquad(n=17) \\
\text { III }\end{array}$ & $\begin{array}{c}\text { Normal control } \\
\text { group } \\
(n=56) \\
V\end{array}$ \\
\hline \multicolumn{5}{|l|}{ Gender $(n)$} \\
\hline Female & 13 & II & 10 & 34 \\
\hline \multicolumn{5}{|l|}{ Age (years) } \\
\hline Mean (s.d.) & $32.8(8.7)$ & $40.7(10.9)$ & $34.0(9.53)$ & $32.2(8.8)$ \\
\hline Range & $21-50$ & $21-52$ & $23-50$ & $21-50$ \\
\hline Smoking $(n)$ & 16 & II & 12 & 39 \\
\hline \multicolumn{5}{|l|}{ DSM-IV diagnosis $(n)$} \\
\hline Schizoaffective disorder & II & 8 & 9 & \\
\hline Paranoid schizophrenia & 10 & 10 & 8 & \\
\hline \multicolumn{5}{|l|}{ Duration of illness (years) } \\
\hline Mean (s.d.) & $7.62(5.2)$ & $6.2(5.4)$ & $5.8(5.03)$ & \\
\hline Range & $2-20$ & $1-18$ & $1-16$ & \\
\hline \multicolumn{5}{|l|}{ Treatment (mg/day) } \\
\hline Mean (s.d.) & $400(94.87)$ & $15.4(14.5)$ & $14.8(4.5)$ & \\
\hline Range & $200-600$ & $5-60$ & $7.5-20$ & \\
\hline
\end{tabular}

units of the spectral components of the HRV before the statistical analysis.

\section{RESULTS}

Demographic data are presented in Table 1 , and electrophysiological results in Table 2 . Patients with schizophrenia taking clozapine had a significantly higher mean heart rate than those taking haloperidol or olanzapine or the control subjects $(P=0.0002)$. As a group, the patients with schizophrenia showed a significantly higher mean heart rate than the matched controls $(P=0.0017)$.

The patients treated with clozapine had significantly lower HRV than those treated with haloperidol or olanzapine or the matched control subjects $(P=0.00017)$. The patient study population showed a significantly lower HRV than the matched controls $(P=0.002)$. The clozapine-treated group had a significantly high low-frequency component compared with patients treated with olanzapine or haloperidol or the control group.

Prolonged QTc intervals were more common in treated patients than in controls although the PR and QRS intervals did not differ significantly. Prolonged QTc intervals were observed in 15 patients treated with clozapine $(71.43 \%), 11$ patients treated with olanzapine $(64.7 \%)$ and 12 patients treated with haloperidol (66.67\%).
A T-wave inversion was observed in fourteen patients treated with clozapine $(66.6 \%)$, three patients treated with olanzapine $(17.6 \%)$, three patients treated with haloperidol $(16.6 \%)$ and in one subject from the control group (1.6\%).

Spontaneous fluctuations in interbeat interval and power spectral analysis of $\mathrm{HRV}$ in treated patients and normal controls are presented in Fig. 1.

There were no significant changes in the respiratory rate (range 13-17 cycles/ min) in any of the subjects. This implies that any significant differences in the highfrequency component can be attributed to non-respiratory effects on parasympathetic tone.

\section{DISCUSSION}

\section{Antipsychotic medication and sudden death}

Common adverse effects of antipsychotic medication include those related to direct or indirect central or peripheral effects on various neurotransmitter and neurohormonal systems (Lader, 1999). In general, psychotic disorders are related to higher rates of death, not all of which can be related causally to medications, including suicide (Chute et al, 1999). Antipsychotic medications have been associated with clinically significant cardiovascular adverse effects and instances 
Table 2 Power spectral analysis of heart rate variability in patients with schizophrenia v. normal controls

\begin{tabular}{|c|c|c|c|c|c|c|c|c|c|}
\hline Power spectral analysis' & \multicolumn{2}{|c|}{$\begin{array}{l}\text { Patients treated } \\
\text { with clozapine } \\
\qquad(n=2 I)\end{array}$} & \multicolumn{2}{|c|}{$\begin{array}{l}\text { Patients treated } \\
\text { with haloperidol } \\
\qquad(n=18)\end{array}$} & \multicolumn{2}{|c|}{$\begin{array}{l}\text { Patients treated } \\
\text { with olanzapine } \\
\qquad(n=17)\end{array}$} & \multicolumn{2}{|c|}{$\begin{array}{c}\text { Normal } \\
\text { control group } \\
(n=56)\end{array}$} & $\begin{array}{c}\text { Scheffé } \\
\text { post hoc } \\
\text { test }\end{array}$ \\
\hline \multicolumn{10}{|l|}{ Time domain } \\
\hline Mean $\mathrm{R}-\mathrm{R}$ interval ${ }^{2}$ & 568.0 & $(14.4)$ & 715.8 & $(33.3)$ & 685.3 & $(23.9)$ & 961.5 & $(84.0)$ & $\mathrm{I} \neq \mathrm{II}, \mathrm{III}, \mathrm{V}$ \\
\hline Standard deviation ${ }^{3}$ & 23.79 & $(3.15)$ & 26.7 & $(2.6)$ & 26.1 & $(4.56)$ & 29.26 & $(6.5)$ & $\mathrm{I} \neq \mathrm{II}, \mathrm{III}, \mathrm{V}$ \\
\hline Variance $^{4}$ & 583.56 & $(14.4)$ & 731.3 & $(33.25)$ & 700.9 & $(23.9)$ & 998.7 & (44.7) & $\mathrm{I} \neq \mathrm{II}, \mathrm{III}, \mathrm{V}$ \\
\hline \multicolumn{10}{|c|}{ Absolute (log) power values of the frequency bands $\left(\mathrm{ms}^{2}\right)$} \\
\hline Low frequency $(L F)^{5}$ & 3.58 & $(0.4)$ & 5.72 & $(0.4)$ & 5.98 & $3(0.34)$ & 4.6 & $(1.35)$ & $\mathrm{I} \neq \mathrm{II}, \mathrm{III}, \mathrm{V}$ \\
\hline High frequency $(\mathrm{HF})^{6}$ & 2.32 & $(0.44)$ & 5.56 & $(0.37)$ & 5.8 & $(3.6)$ & 4.6 & $(1.32)$ & $\mathrm{I} \neq \mathrm{II}, \mathrm{III}, \mathrm{V}$ \\
\hline Heart rate ${ }^{7}$ (b.p.m.) & 107 & $(2.7)$ & 85.8 & (3.89) & 89.05 & $(3.08)$ & 62 & $(12.4)$ & $\mathrm{I} \neq \mathrm{II}, \mathrm{III}, \mathrm{V}$ \\
\hline $\mathrm{QT}^{8}$ & 518.3 & (II.5) & 475 & $(6.4)$ & 474.3 & $(8.02)$ & 345.2 & $(5.0)$ & \\
\hline QTc ${ }^{9}$ & 502.9 & $(12)$ & 517.8 & $(15.08)$ & 504.6 & $(7.0)$ & 370 & $(12.2)$ & \\
\hline QRS $(\mathrm{ms})^{10}$ & 94.5 & $(18.5)$ & 92.2 & $(19.3)$ & 95.5 & $(14.3)$ & 92.1 & $(18.2)$ & NS \\
\hline PR (ms) ${ }^{\prime \prime}$ & 150.2 & $(26.3)$ & 148.2 & $(24.2)$ & 152.1 & $(22.3)$ & I5I.I & (20.4) & NS \\
\hline \multicolumn{10}{|l|}{ Frequency domain } \\
\hline \multicolumn{10}{|l|}{ Power } \\
\hline$L F(\%)^{12}$ & 74.2 & $(3.46)$ & 53.8 & $(2.75)$ & 54.1 & $(2.74)$ & 49.0 & $(1.5)$ & $\mathrm{I} \neq \mathrm{II}, \mathrm{III}, \mathrm{V}$ \\
\hline $\mathrm{HF}(\%)^{13}$ & 25.8 & $(3.46)$ & 46.2 & $(2.75)$ & 45.9 & $(2.74)$ & 51.0 & $(1.5)$ & $\mathrm{I} \neq \mathrm{II}, \mathrm{III}, \mathrm{V}$ \\
\hline $\mathrm{LF} / \mathrm{HF}(\%)^{14}$ (mean of individual ratios) & 5.9 & $(1.88)$ & 1.28 & $3(0.18)$ & 1.32 & $(0.18)$ & 0.96 & $(0.03)$ & $\mathbf{I} \neq \mathbf{V}$ \\
\hline
\end{tabular}

I. Results are expressed as in mean (s.d.) in normalised units using one-way ANCOVA and the covariates of age and smoking.

2. $F=77.27, P<0.0001$

3. $F=72.7, P<0.0001$

4. $F=66.7, P<0.0001$

5. $F=11.3, P<0.0001$

6. $F=21.7, P<0.0001$.

7. $F=63.08, P<0.0001$.

8. $F=10.469, P<0.0001$.

9. $F=10.469, P<0.0001$.

10. $F=10.469, P<0.0001$

II. $F=10.469, P<0.0001$

12. $F=19.84, P<0.0001$.

13. $F=19.84, P<0.0001$

14. $F=4.5, P=0.0064$.

of sudden death. Controversy continues to surround the issue of sudden death in patients with schizophrenia (Anon., 1966), although numerous reports of sudden deaths of patients on antipsychotic medication exist and many authors relate the phenomenon to medications (Ruschena et al, 1998; Chute et al, 1999).

The possible association between sudden death and antipsychotic pharmacotherapy has been reviewed most extensively by a task force of the American Psychiatric Association, whose disappointingly vague conclusion is that: "the role played by the drug in a given individual is difficult, if not impossible, to determine" (Task Force, 1988). Despite the inconclusive results of the task force, the possibility of arrythmogenic properties of antipsychotic drugs cannot be excluded.

\section{Association of autonomic dysfunction and clozapine}

The results of this study provide evidence that power spectral analysis of HRV may be of value in assessing the effects of medications on the physiological parameters of patients. Patients with schizophrenia under prolonged clozapine therapy exhibited marked differences in ANS functioning compared with those treated with haloperidol or olanzapine, as shown by increased heart rate and low-frequency components and lower HRV and high-frequency components. This reflects a basal autonomic dysfunction with increased sympathetic and decreased parasympathetic tone. Our findings are in complete agreement with those of Zahn \& Pickar (1993) and Rechlin et al (1998), who demonstrated normal
HRV in unmedicated patients with schizophrenia and a dose-related effect of clozapine on HRV. We thus attribute these findings to clozapine and not to the disease.

The difference between the effects of clozapine, olanzapine and haloperidol on HRV is very interesting. On the one hand, haloperidol is known to be relatively free of clinically significant effects on receptors for neurotransmitters other than dopamine (except for a low affinity for $\alpha_{2^{-}}$and 5$\mathrm{HT}_{2 \mathrm{a}}$-receptors), whereas both clozapine and olanzapine have more wide-ranging receptor affinity profiles of clinical significance (Shiloh et al, 1999). On the other hand, the findings of this study show a greater in vivo similarity between the HRV effects (i.e. clinical ANS effects) of olanzapine and haloperidol than those of clozapine. This clinical finding is difficult to explain in 

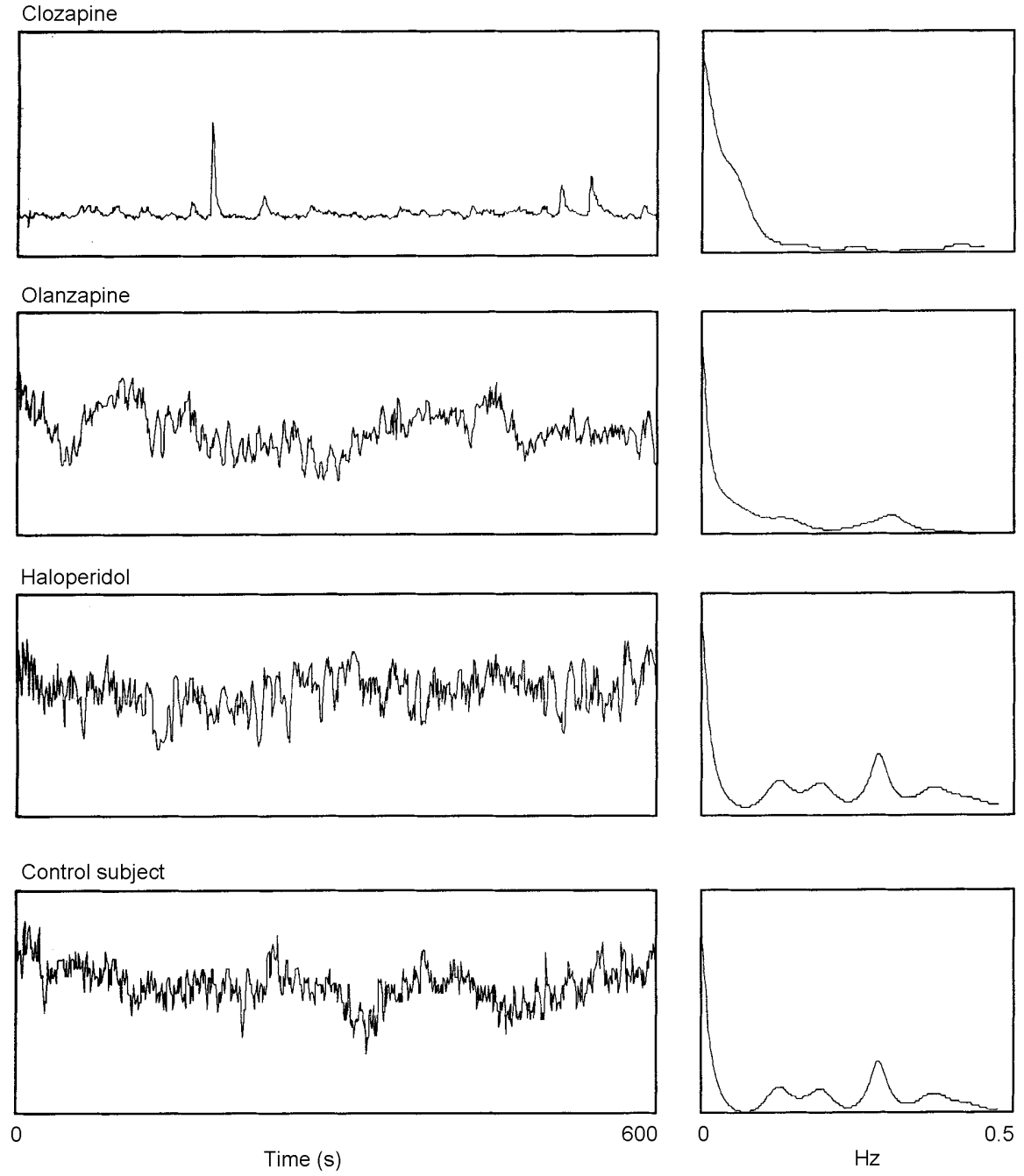

Fig. I (Left) Spontaneous fluctuations in interbeat interval of a 600-s time period during supine rest and (right) power spectral analysis of HRV in patients with schizophrenia and normal controls. The power spectra show the HRV in all frequency bands ( $y$-axis: $\mathrm{ms}^{2} / 10^{3} \mathrm{~Hz}$ ).

terms of a simple neurotransmitter/receptor paradigm and is probably the net result of complex interactions between peripheral and central mechanisms (Jacobs, 1987).

\section{Antipsychotics and QT interval}

Some of the effects of antipsychotic medications appear to be direct and local. A high prevalence of QTc prolongation was found in all our treated patients compared with controls, a finding consistent with the trend of the findings in other clinical reports (Barnett, 1996; Reilly et al, 2000). The high prevalence of QTc prolongation is in keeping with the trend of the findings reported by Reilly et al (2000), although the proportion of patients with QTc prolongation is far greater in our study. Preclinical (animal) studies also support this trend (Drici et al, 1998). The PR and QRS intervals did not differ significantly. This probably suggests that neuroleptics affect cardiac repolarisation rather than conduction, owing to their sodium/potassium as well as calcium-ionchannel blocking actions. Drugs that significantly prolong the QT interval may have the potential to trigger complex forms of polymorphic ventricular arrhythmias (e.g. torsade de points), which can lead to sudden death.

Our findings imply that patients with schizophrenia taking clozapine have decreased parasympathetic activity, elevated sympathetic activity and prolonged QT intervals. Because decreased parasympathetic tone lowers the threshold for ventricular tachycardia (Suttmann et al, 2000), it is possible, although as yet unproven, that the decreased high-frequency component may reflect the mechanism that underlies the increased rate of cardiovascular mortality in patients with schizophrenia.

\section{Possible mechanisms by which neuroleptic medications cause malignant cardiac arrhythmias}

The precise cellular mechanisms by which neuroleptic medications cause malignant cardiac arrhythmias are poorly understood. The triggering mechanism is thought to be the interruption of repolarisation early in phase 3 of the action potential by early after-depolarisations (Drici et al, 1998). Early after-depolarisations are induced by interventions that decrease repolarising (potassium) currents (antiarrhythmic drugs) and/or that increase inward currents carried by calcium or sodium (January et al, 1988; Roden et al, 1996). These changes may produce early after-depolarisations directly, or they may alter the trajectory of the antipsychotic to produce secondary changes in otherwise normal ion currents that then result in early after-depolarisations. These early after-depolarisations may reach a threshold amplitude and trigger ventricular arrhythmias (Viskin, 1999). Additionally, the QT prolongation, resulting from the blockade of one or more repolarising potassium channels (Viskin, 1999), may cause a surplus of potassium ions in the intracellular volume of the myocardium, enabling the genesis of early after-depolarisations. This phenomenon is seen in the 'long-QT syndrome', which is characterised by similar features and is associated with fatal arrhythmias, presumably secondary to the attainment of depolarisation threshold. Autonomic dysregulation also has been associated with arrhythmic effects in otherwise healthy individuals, as the result of sympathetic hyperactivity and/or parasympathetic hypoactivity. The emergence of torsade de pointes also may be facilitated by electrolyte disturbances (hypokalaemia, hyponatraemia, hypomagnesaemia), secondary to clinical or subclinical syndrome of inappropriate antidiuretic hormone secretion (SIADH), a rare toxic effect of neuroleptic medication. Independent risk factors, such as smoking, may contribute.

Clinical experience has shown that many patients with schizophrenia require long-term pharmacotherapy, and the reports of a possible association between antipsychotic drugs and sudden death cause concern for practitioners. Our present experience in applying power spectral analysis of HRV as an index of cardiac autonomic balance is rather encouraging. The method is non-invasive, relatively inexpensive, straightforward and uncomplicated to operate and provides a real-time 
dynamic assessment of sympathetic and parasympathetic tone, reflecting the interactions between the two. This study raises the question of potentially important prognostic implications of protracted autonomic dysfunction in medicated psychiatric patient populations, especially for cardiovascular morbidity and mortality. Diagnostic measurement of the ANS may improve identification of patients at high risk for sudden cardiac death. Measurement of heart rate and QT are far less specific than power spectral analysis of HRV. Heart rate is affected by too many non-specific factors and QT changes are near-universal effects of neuroleptic medication, whereas HRV clearly reflects ANS pathophysiology.

The results of this study raise the possibility that the cardiovascular effects of neuroleptics may, at least in part, be mediated by autonomic dysregulation, in addition to other simultaneous effects accounting for the cardiac cellular electrophysiological level. Prospective studies may identify electrocardiographic markers of cardiac damage in at-risk patients, which may be useful in screening for these important adverse outcomes.

\section{REFERENCES}

Akselrod, S., Gordon, D., Ubel, F., et al (198I) Power spectral analysis of heart rate fluctuation: a quantitative probe of beat-to beat cardiovascular control. Science, 213, 220-222.

American Psychiatric Association (1994) Diagnostic and Statistical Manual of Mental Disorders (4th edn) (DSM-IV). Washington, DC: APA

Anon. (1966) Sudden death and phenothiazines. Lancet, ii, 740 .

Appleby, L., Thomas, S., Ferrier, N., et al (2000)

Sudden unexplained death in psychiatric in-patients. British Journal of Psychiatry, 176, 405-406.

Barnett, A. (1996) Safety concerns over antipsychotic drug: sertindole. Lancet, 348, 256

Chute, D., Grove, C., Rajasekhara, B., et al (1999) Schizophrenia and sudden death: a medical examiner case study. American Journal of Forensic Medical Pathology, 20, $|3|-\mid 35$.

Drici, M. D., Wang, W. X., Liu, X. K., et al (1998) Prolongation of QT interval in isolated feline hearts by antipsychotic drugs. Journal of Clinical Psychopharmacology, I8, 477-48I.

Endicott, J. \& Spitzer, R. L. (1987) Schedule for Affective Disorders and Schizophrenia (SADS). Acta Psychiatrica Belgica, 87, 36I-516.

Jacobs, B. (1987) Central Monoaminergic Neurons: Single-Unit Studies in Behaving Animals. New York: Raven Press.

January, C. T., Riddle, J. M. \& Salata, J. J. (1988) A model for early afterdepolarizations: induction with

\section{CLINICAL IMPLICATIONS}

- Power spectral analysis of heart rate variability (HRV) demonstrates an effect of antipsychotic medications on autonomic regulation, in addition to the cellular electrophysiological effects described elsewhere.

- The study emphasised the usefulness and relatively simple application of power spectral analysis of HRV as a tool for research and possibly 'bedside' assessment of the autonomic and cardiovascular effects of medications, being simple, non-invasive and reliable.

- Power spectral analysis of HRV demonstrated effects of antipsychotic medications not directly explicable on the basis of receptor affinity profiles and thus raises questions regarding mechanisms of action, which warrant further study.

\section{LIMITATIONS}

- The study group was small as a result of inclusion requirements such as monotherapy and absence of associated diseases.

- The findings relate to only three medications, because we sought to limit the study to patients on neuroleptic monotherapy, and must be extended to involve a broader range of medications.

- The results thus must be regarded as preliminary, although of significant clinical importance in their possible implications, regarding the need to focus research efforts on the possible cardiovascular complications of neuroleptics.

HAGIT COHEN, PhD, URI LOEWENTHAL, MD, MICHAEL MATAR, MD, MOSHE KOTLER, MD, Ministry of Health Mental Health Center, Faculty of Health-Sciences, Anxiety and Stress Research Unit, Ben-Gurion University of the Negev, Beer-Sheva, Israel

Correspondence: Hagit Cohen, Anxiety and Stress Research Unit, Ministry of Health Mental Health Center, Faculty of Health-Sciences, Ben-Gurion University of the Negev, PO Box 4600, Beer-Sheva, Israel. Fax: 972-8-6401742; e-mail: hagitc@bgumail.bgu.ac.il

(First received II August 2000, final revision 22 February 200I, accepted 7 March 200I)

the $\mathrm{Ca}^{2+}$ channel agonist Bay K 8644. Circulation Research, 62 , 563-571.

Lader, M. (1999) Some adverse effects of antipsychotics: prevention and treatment. Journal of Clinical Psychiatry, 60 (suppl. 12), 18-2I.

Rechlin, T., Beck, G., Weis, M., et al (1998) Correlation between plasma clozapine concentration and heart rate variability in schizophrenic patients. Psychopharmacology (Berlin) I35, 338-34!

Reilly, J. G., Ayis, S. A., Ferrier, I. N., et al (2000) QT c-interval abnormalities and psychotropic drug therapy in psychiatric patients. Lancet, 355, 1048-1052.

Roden, D. M., Lazzara, R., Rosen, M., et al (1996) Multiple mechanisms in the long-QT syndrome.Current knowledge, gaps, and future directions. The SADS FoundationTask Force on LQTS. Circulation, 94, 1996-2012.

Ruschena, D., Mullen, P. E., Burgess, P., et al (1998) Sudden death in psychiatric patients. British journal of Psychiatry, 172, 331-336.
Shiloh, R., Nutt, D. \& Weizman, A. (1999) Atlas of Psychiatric Pharmacotherapy (Ist edn). London: Martin Dunitz.

Suttmann, I., Ditter, S., Landgraf, R., et al (2000) Clozapine and sudden death. Lancet, 355, 842-843.

Task Force (1988) Sudden Death in Psychiatry Patients: the Role of Neuroleptic Drugs. Washington, DC: American Psychiatric Association.

- (1996) Heart rate variability. Standards of measurement, physiological interpretation, and clinical use. Task Force of the European Society of Cardiology and the North American Society of Pacing and Electrophysiology. European Heart Journal, 17, 354-38I.

Viskin, S. (1999) Long QT syndromes and torsade de pointes. Lancet, 354, 1625-1633.

Zahn, T. P. \& Pickar, D. (1993) Autonomic effects of clozapine in schizophrenia: comparison with placebo and fluphenazine. Biological Psychiatry, 34, 3-12. 